2015-12-31

\title{
Playing Tetris decreases drug and other cravings in real world settings
}

Skorka-Brown, J

http://hdl.handle.net/10026.1/3458

10.1016/j.addbeh.2015.07.020

Addictive Behaviors

Elsevier BV

All content in PEARL is protected by copyright law. Author manuscripts are made available in accordance with publisher policies. Please cite only the published version using the details provided on the item record or document. In the absence of an open licence (e.g. Creative Commons), permissions for further reuse of content should be sought from the publisher or author. 
This is a pre-refereeing authors' version of a manuscript that is now published as

Skorka-Brown, J., Andrade, J., Whalley, B. \& May, J. (in press, July 2015). Playing Tetris decreases drug and other cravings in real world settings. Addictive Behaviors.

Playing Tetris decreases drug and other cravings in real world settings

$$
\begin{gathered}
\text { Jessica Skorka-Brown }^{1} \\
\text { Jackie Andrade }^{1,2} \\
\text { Ben Whalley }^{1} \\
\text { Jon May } \\
\end{gathered}
$$

${ }^{1}$ School of Psychology, Cognition Institute, Plymouth University, UK

${ }^{2}$ Institute of Health and Biomedical Innovation and School of Psychology and Counselling, Queensland University of Technology, Australia

Address for correspondence: Jackie Andrade, School of Psychology, Cognition Institute, Plymouth University, Drake Circus, Plymouth PL4 8AA, UK; email jackie.andrade@plymouth.ac.uk; Tel. +44 1752584807

Running head: Tetris decreases craving Acknowledgements: This research was supported by a PhD studentship awarded to Jessica Skorka-Brown by the School of Psychology. We would like to thank Kishan 
Gnanasekaran for his help with data collection and Lynne James for all her hard work with the programming.

Keywords: Desire; cognitive theory; behavioral research; Elaborated Intrusion theory; addiction; technology; multilevel models

Word count: body excluding references $=3399$ words

Highlights:

- Playing Tetris weakened cravings in natural settings

- Tetris was effective for drug cravings as well as food and activity cravings

- Effect was consistent over a week

- Tetris was effective on higher cravings experienced under influence of alcohol 


\begin{abstract}
Most research on cognitive processes in craving has been carried out in the laboratory and focuses on food craving. This study extends laboratory findings to real world settings and cravings for drugs or activities as well as food. Previous laboratory research has found that playing Tetris reduces craving strength. The present study used an ecological momentary assessment protocol in which 31 undergraduate participants carried iPods for a week and were prompted 7 times each day, by SMS message, to use their iPod to report craving. Participants reported craving target and strength (0100), whether they indulged their previous craving (yes/no), and whether they were under the influence of alcohol (yes/no). Those randomly assigned to the intervention condition $(n=15)$ then played Tetris for 3 minutes and reported their craving again. Those in the monitoring-only control condition $(n=16)$ provided baseline craving data to test if Tetris reduced the incidence and strength of spontaneous cravings across the week. Playing Tetris decreased craving strength for drugs (alcohol, nicotine, caffeine), food and drink, and activities (sex, exercise, gaming), with a mean reduction of 13 percentage points, effect size $\mathrm{f}^{2}=0.11$. This effect was consistent across the week. This is the first demonstration that visual cognitive interference can be used in the field to reduce cravings for substances and activities other than eating.
\end{abstract}

216 Words 
Do you ever find yourself thinking about a big mug of steaming-hot coffee and finding it hard to concentrate on work until you have one? Hofmann, Baumeister, Forster and Vohs (2011) showed that cravings like this are a common occurrence. On almost $50 \%$ of occasions sampled, participants reported a current craving, and just under half of these cravings caused conflict for the person experiencing them. An effective strategy for tackling cravings could have a large impact on a person's life particularly for someone with an addiction who is trying to reduce or quit drug use.

Craving is listed as a feature of substance dependence in the International Classification of Diseases (ICD-10; World Health Organisation, 1992), and has been introduced as a criterion for substance use disorders in DSM-V (APA, 2013). It is clinically significant (Tiffany \& Wray, 2012), contributing to continuation of drug use (Piaseki, Richardson \& Smith, 2007) and relapse after quitting (Shiffman et al., 1997). Elaborated Intrusion (EI) theory (Kavanagh, Andrade \& May, 2005) explains the cognitive processes underpinning craving, and assumes these processes operate across cravings for different substances and activities. Psychological strategies for managing cravings should also act across diverse cravings. This paper reports the first test of this prediction in a naturalistic setting, with craving targets including addictive substances, food and drink, and physical activities.

In El theory, cravings are viewed as cognitive-emotional states involving embodied sensory imagery in the same modalities as the desired substance or activity. Motivation to use a drug or consume food is driven by the imagined experience of achieving that goal. El theory is supported by evidence on phenomenology of cravings, where sensory imagery features in cravings for a range of addictive and everyday targets, including 
alcohol (Kavanagh, May \& Andrade, 2009; Statham, Connor, Kavanagh, et al., 2012, May, Andrade, Kavanagh, et al., 2014), food (Harvey, Kemps \& Tiggemann, 2005; May, Andrade, Panabokke \& Kavanagh, 2004; May, Andrade, Kavanagh \& Penfound, 2008; May et al., 2014), cigarettes (May et al., 2004; Salkovskis \& Reynolds, 1994; May et al., 2014), and sport (Hall, Rodgers, Wilson, \& Norman, 2010; May et al., 2008). Craving imagery is associated with behaviour, for example with alcohol use (Connor, Kavanagh, Andrade, et al., 2014) and exercise frequency (Gammage, Hall, \& Rogers, 2000).

Imagery places demands on limited-capacity working memory processes (Baddeley \& Andrade, 2000). Consistent with the hypothesis that imagery plays a role in craving, laboratory research has shown that cravings can be suppressed by a working memory load (Van Dillen, Papies, \& Hofmann, 2013). Tasks that involve visuospatial or olfactory processing tend to be more effective than auditory tasks across a range of targets, including cigarettes (May, Andrade, Panabokke \& Kavanagh, 2010, Versland \& Rosenberg, 2007), food (Andrade, Pears, May \& Kavanagh, 2012; Kemps \& Tiggemann, 2007), and coffee (Kemps \& Tiggemann, 2009), supporting the assumption that craving involves sensory imagery in the same modalities as the desired activity.

These laboratory findings point the way to new interventions for tackling problematic cravings. Cravings should be reduced by cognitive tasks that target the working memory processes that support craving imagery. Visual imagery should be a good target for a broad range of cravings as relevant studies to date have shown a role for visual imagery in all cravings studied (May et al., 2014). 
Three studies have tested the impact of El theory-based interventions on food craving in field settings. Kemps and Tiggemann (2013) used paper diaries to measure craving, and a passive visual interference task called dynamic visual noise to interfere with it. Dynamic visual noise reduces craving for cigarettes (May et al., 2010, experiment 2) and food (Kemps, Tiggemann \& Christianson, 2008) in the laboratory. Kemps and Tiggemann (2013) found that it also reduced food craving in the field. Knäuper, Pillay, Lacaille, McCollam and Kelso (2011) recruited participants who reported strong cravings for specific food or drinks (commonly chocolate and coffee) and wanted to reduce those cravings. As predicted by El theory, participants who vividly imagined a favourite activity whenever cravings struck reported weaker cravings than those who recited the alphabet backwards. Hsu, Yang, Yilmaz,, Hague, Can \& Blandford (2014) built a mobile application, iCrave, based on El theory, which prompted vivid, neutral imagery. Participants who used the app reported less snacking and greater ease of abstaining that those who simply used an app to track their snacking.

Laboratory studies suggest that visuospatial tasks will also block drug cravings, with a few studies showing reductions in cravings for cigarettes (May et al., 2010; May, Andrade, Willoughby \& Brown, 2012; Versland \& Rosenberg, 2007) and caffeine (Kemps \& Tiggemann, 2009), and one study of naturally-occurring cravings that included cravings for caffeine and cigarettes (Skorka-Brown, Andrade \& May, 2014).

The present study extends this research to a wider range of cravings, including drug cravings, to test if competing visuospatial loads reduce craving strength and decrease indulgence of cravings in naturalistic settings. Rather than rely on participants to complete a task spontaneously, as previous studies have done, we used an ecological 
momentary assessment protocol with SMS messages to prompt reporting of craving and engagement with the intervention task on portable devices (iPods) carried by participants. We used Tetris as the intervention task because it is an easily accessible, engaging task with a strong visual component including brightly coloured shapes, movement, and mental rotation. Tetris blocks development of distressing intrusive images (Holmes, James, Coode-Bate \& Deeprose, 2009; Holmes, James, Kilford \& Deeprose, 2010) and weakens naturally occurring cravings in a laboratory setting (Skorka-Brown et al., 2014). Additionally, participants were asked if they were under the influence of alcohol (including being hungover) as previous research (Burton \& Tiffany, 1997) has shown that participants experienced an increase in cravings when they had consumed alcohol. It is not known whether these cravings are more or less resistant to cognitive interference.

\subsection{Method}

\subsubsection{Participants and Design}

A total of 31 (7 males) undergraduate psychology students took part in partial fulfilment of a course requirement to participate in research. They were aged between 18 and 27 years $(m=20.6 S D=2.3)$. Participants were randomly assigned to either 'Tetris' or control (monitoring only) conditions, (16 Control, 15 Tetris).

\subsubsection{Materials}

Two different models of iPod Touch were used (first and second generation models, manufactured by Apple), both with a 3.5 inch multi-touch display. The program was 
written in Objective-C, and utilised the Cocos2D library. Participants used these iPods in response to SMS prompts sent to their mobile phones to access a questionnaire and, depending upon condition, a game of Tetris.

In the Control condition, participants completed a questionnaire about craving following each prompt. Participants were asked 'Have you indulged in the item you reported craving previously?' (response options: yes/no/was not craving previously); how much are you under the influence of alcohol? (five-point scale, from not at all to very much); are you currently craving anything? (yes/no). If they responded yes to the current craving question, they were asked to report what they were craving and how strongly they were craving it (0-100 scale). This control group served to show how craving varied during the study week, allowing us to test if playing Tetris ultimately reduced the tendency to experience cravings or weakened cravings that did occur.

In the Tetris condition, participants completed the same questionnaire and in addition then played Tetris for 3 minutes, manipulating coloured basic shapes to form rows that would then disappear allowing for game play to continue; if the screen became full, a new game started. Participants played Tetris even if they had reported no craving. After 3 minutes, participants re-stated their craving strength for the same item, on the same 0-100 point scale.

A retrospective measure of the strongest episode of craving over the prior week was obtained at the start and end of the study period using the strength form of the Craving Experience Questionnaire (CEQ; May et al., 2014), with the instruction 'Think about the time you most craved something during the past week'. The 11-item CEQ asks 
participants to rate their craving on three factors derived from Elaborated Intrusion theory: intensity (e.g., How much did you want it?), imagery (e.g., How vividly did you picture it?), and intrusiveness (e.g., How intrusive were the thoughts?). With the exception of two questions that asked what the person had most craved over the past week, and how long the craving had lasted, items were rated on a scale of 0 (not at all) to 10 (extremely). May et al. (2014) reported a high coefficient alpha of .91 for the scale, consistent factor structure across substances, and robust associations with other measures of desire and dependency.

\subsubsection{Procedure}

Participants came to the laboratory on a Wednesday morning and completed a short demographics questionnaire, the CEQ and were shown how to use the iPod.

Participants were asked to complete their iPod task whenever they received a SMS message prompting them to do so. They were given written instructions to take away with them.

Participants carried the iPod with them for the week. They were texted on a pseudorandom sampling schedule. They received 7 texts each day between the hours of 9am and 10pm. The day was broken into 7 sections (9am-10.30, 10.30-12.30, 12.30-2.30, 2.30-4.30, 4.30-6.30, 6.30-8.30, 8.30-10pm) and participants were texted at one random time within each section, but not within the final 30 minutes of a section, to allow participants the minimum of 30 minutes to do the task. The text they received gave a deadline for completing the task, of 30-60 minutes depending on proximity to the next 
time section. Participants were told that it was important to complete the task within the specified timeslot. The iPod recorded the time that the task was done.

Participants returned to the laboratory the following Tuesday late afternoon to return their iPod and complete the CEQ. Participants missed a total of 6 prompts on the first and last day of the study due to the timings of these sessions.

\subsubsection{Statistical methods}

Stata 13 (StataCorp, 2013) was used to analyse craving strength data by mixed-effects regression, where craving scores were nested within individuals.

The primary analysis, using data from the Tetris group only, tested the hypothesis that playing Tetris would reduce craving. Our model included fixed effects to estimate the difference in craving strength before and after game completion. We further tested whether this effect altered over the week. Estimates of the effect of playing Tetris were stratified (using interaction terms) by the type of craving reported, and whether the participant was under the influence of alcohol.

Data from the control condition allowed us to test whether Tetris chronically reduced the incidence and strength of cravings, by comparing the probability of craving and craving strength after each initial prompt (for the Tetris group, this was the response made before playing the game) between participants in the Tetris and control conditions.

Indulgence was recorded as a binary variable indicating whether or not the participant had indulged their last-reported craving, and analysed using mixed-effects regression with a logit link function. 


\subsection{Results}

Participants were deemed compliant if they completed the iPod task within the allotted time. Out of 43 prompts, participants responded to an average of $34.3(\mathrm{SD}=7.50$; all but one responded to at least 23 prompts). At $80 \%$, this level of compliance is similar to rates reported elsewhere (e.g. Hofmann, Baumeister, Forster \& Vohs 2011; Waters \& Li, 2008).

To test the effect of Tetris on craving strength, our mixed model regressed reports of craving strength on measurement-point (before/after Tetris) and day. Model estimates of craving strength were on average 13.06 points lower $(95 \% \mathrm{Cl}=-20.1$ to -4.7$)$ after playing Tetris $($ marginal mean $=57.58)$ than before playing $($ marginal mean $=70.64) \mathrm{a}$ medium effect size of $f^{2}=0.11$. This effect did not vary over the week $x^{2}(6)=1.98, p=$ 0.921 (Figure 13). 


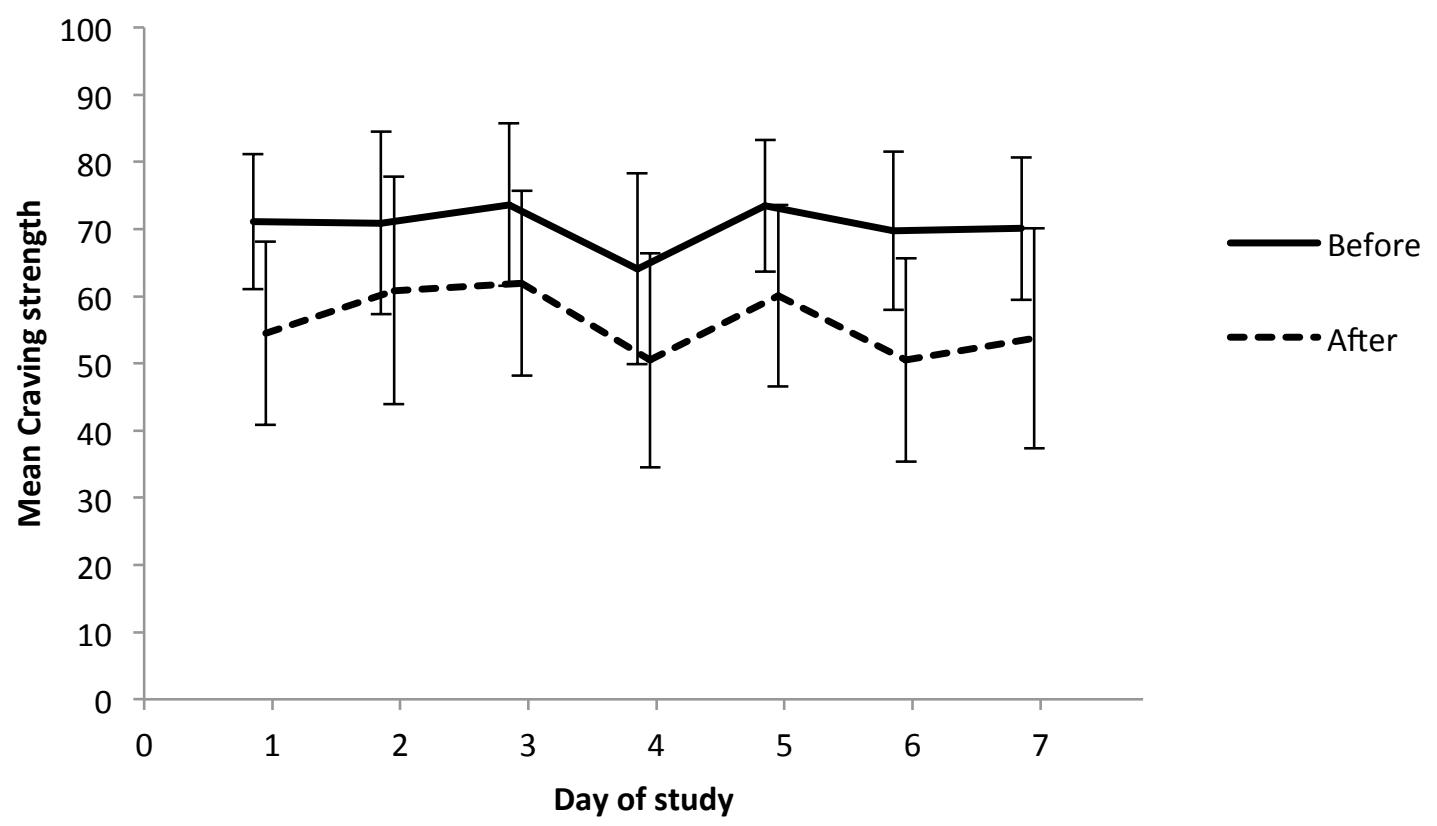

Figure 1: Effect of playing Tetris on craving strength across the week. Solid line shows mean ratings before playing, dashed line shows mean ratings after playing Tetris. Error bars show $95 \% \mathrm{Cl}$.

We examined the strength of different types of craving before and after playing Tetris.

There was a trend for types of craving to differ in strength, $X^{2}(2)=5.67, p=0.059$ (see Table 1). Pairwise comparisons showed that cravings for addictive substances (nicotine, alcohol, caffeine) were reported as significantly more intense than cravings for food or drink $\left(\Delta=6.245, \chi^{2}(1)=4.28, p=0.039\right)$, but not stronger than cravings for the 'other' category, which included exercise, sex, and gaming $\left(\Delta=0.650, x^{2}(1)=0.03, p=0.864\right)$. The interaction between measurement point (before/after playing Tetris) and type of craving was not significant, $x^{2}(2)=0.98, p=0.611$; this suggests that the effect of playing Tetris was similar across the different craving types (Table 1). 
Table 1 . Mean craving strength $( \pm 95 \%$ confidence interval) by craved substance or activity in the Monitoring Only control condition and in the experimental condition before and after playing Tetris.

$\begin{array}{llll}\text { Craving Target } \quad \text { Monitoring Only } & \text { Before Tetris } & \text { After Tetris }\end{array}$

Addictive

Substances

$70.54(61.59-79.49) \quad 73.14(64.60-81.67) \quad 59.71(51.18-68.24)$

Food/Drink $\quad 67.93(61.48-74.38) \quad 67.80(60.97-74.64) \quad 52.55(45.71-59.39)$

Other

$72.44(62.78-82.09) \quad 70.53(61.03-80.02)$

$61.01(51.52-70.51)$

Mean Across

Craving Types

$$
69.08(62.92-75.25) \quad 69.48(63.12-75.84) \quad 55.59(49.23-61.94)
$$

The influence of alcohol, dichotomised as none vs. any influence, did not predict craving strength (none $=71.56, S D=20.25$ versus any=70.48, $S D=22.48 ; X^{2}(1)=1.08, p=.298$ ). Furthermore, participants under the influence of alcohol benefited from playing Tetris as much of those who were not: There was no interaction between measurement-point (before/after) and influence of alcohol, $x^{2}(1)=2.23, p=0.136$, although our 1063 observations included only 67 when participants were mildly influenced by alcohol and 44 when participants were more than mildly influenced.

Initial craving strength (i.e., the pre-game measurement in the Tetris condition) did not differ between the Tetris and control conditions $(t=0.822, d f=318, p=0.206$, one-tailed, 
equal variances assumed), either overall or within each craved item category (see Table 1 ; smallest $p=0.285$, equal variances assumed). There was no effect of Tetris on initial craving strength or incidence of craving across the week: Using two mixed effects models, we first regressed initial craving strength scores and then craving incidence (initial strength 0 or $>0$ ), on day and condition and their interaction. There was no interaction between day and condition on initial craving strength, $x^{2}(1)=1.29, p=0.255$, nor on incidence of cravings across the week, $x^{2}(1)=1.54, p=0.214$.

Playing Tetris did not appear to reduce people's tendency to indulge their cravings. Multilevel logistic regression found no difference in indulgence in the Tetris compared with the control condition ( $58 \%$ versus $\left.56 \%, \chi^{2}(1)=1.19, p=0.276\right)$. However, participants under the influence of alcohol were more likely to indulge than those who were not $\left(78 \%\right.$ vs. $\left.55 \%, X^{2}(1)=4.04, p=0.044\right)$. There was no interaction between condition and the influence of alcohol, $x^{2}(1)=1.43, p=0.232$.

The type of craving reported was a predictor of subsequent indulgence, $x^{2}(2)=6.61$, $p=0.037$. The probability of participants indulging was .50 if their craving was for food or drink, .63 if their craving was for an addictive substance, and .74 if their craving was in the other category. There was again no effect of condition $x^{2}(1)=0.03, p=0.859$, or interaction with craving type, $x^{2}(2)=1.46, p=0.482$.

ANOVA showed that scores on the CEQ, measuring strength, intrusiveness and vividness of strongest craving in the past week, did not vary with time (baseline, follow up) or condition (control, Tetris; smallest $p=0.055$ ). 


\section{Discussion}

Playing Tetris for three minutes decreased craving strength for addictive substances (nicotine, caffeine and alcohol), food and drink, and other cravings (e.g., sex, gaming, exercise and social interaction) by nearly one fifth throughout the seven-day study period. This is the first demonstration that visual cognitive interference can be used repeatedly in the field to reduce cravings for a range of substances and activities other than food. This finding extends that of Skorka-Brown et al. (2014), who reported that craving strength was reduced by a similar amount (14 points using the same 100 point scale) when participants played Tetris in the laboratory, but not when they watched a fake loading-screen. Using a working memory task to interfere with cravings was as effective in the field as in the laboratory.

Previous studies have shown that craving reduction effects of visuospatial tasks such as neutral visual imagery (Kemps \& Tiggemann, 2007) and dynamic visual noise (Kemps et al., 2008) translate to field settings when food craving is the target (Knäuper et al., 2011; Kemps \& Tiggemann, 2013). Our findings suggest that the effectiveness of these tasks on nicotine cravings in the laboratory (May, et al., 2010, Versland \& Rosenberg, 2007) will also generalise to field settings and other drug targets.

The impact of Tetris on craving did not vary across the week. This finding is important because an intervention that worked solely because it was novel and unusual would have diminishing benefits over time as participants became familiar with it. Tetris did not reduce the initial craving scores across the week. This finding was unsurprising as participants were prompted to play Tetris at specific times, so would be unlikely to have 
played it for all their cravings. Longer-term studies, with participants for whom cravings are unwanted, are needed to test if a tool for managing cravings helps to reduce the incidence and strength of all cravings or, as in the present study, only weakens cravings while it is being used.

There was no effect of Tetris on indulgence rates compared to the control condition. Previous research (Shiffman et al., 2002; 2013) has clearly shown that people report cravings to be related to indulgence, but there are several reasons why the relationship is weaker in this study. First, participants were asked to recall whether they indulged in their craving on the subsequent prompt, after a delay. Recall was sometimes inaccurate, with some participants stating that they had not been craving despite previously reporting a craving. Future research could require indulgences to be reported as soon as they occur, without requiring a subsequent prompt.

Second, indulgence focused on consumption of the craved item or activity, rather than indulgence generally. Knäuper et al. (2011) also focused on specific consumption and found no effect of task on indulgence. In contrast, Kemps and Tiggemann (2013) and Hsu et al. (2014) found that visual interventions decreased general consumption. This may be an important difference that future research could address by measuring both general consumption and specific consumption. We did not measure the amount by which people indulged their craving; it is conceivable that playing Tetris reduced the amount indulged (e.g., the quantity eaten or smoked), rather than stopping the indulgence altogether. 
Third, this study recruited an unrestricted sample whereas Kemps and Tiggemann (2013), Hsu et al. (2014) and Knäuper et al. (2011) recruited participants who had a high number of food cravings, wanted to reduce their snacking behavior, or wanted to reduce their cravings. Those populations may have had stronger motivation to decrease their indulgence rates whereas our sample may have been contented with their indulgence rates and still have chosen to indulge even when their craving was weakened. Wray, Gass and Tiffany's (2013) meta-analysis of relationships between pre-quit craving, post-quit craving and relapse in 62 smoking cessation studies supports this conclusion, showing that post-quit craving scores predict relapse but pre-quit measures do not. It is only when a person is actively trying to resist a substance that decreasing cravings may help decrease consumption.

Participants under the influence of alcohol did not report an increase in craving strength (in contrast to Burton \& Tiffany, 1997) but did have higher indulgence rates. However, cravings under the influence of alcohol occurred for only just over $10 \%$ of prompts, so these results may not be representative. The timeslots used to spread out prompts during the day meant that participants only received one prompt after $8.30 \mathrm{pm}$ each day, which is when drinking is most likely in the student population that was sampled. Future research could obtain more reports of craving later in the evening, to obtain a better estimate of any interactions with alcohol consumption.

Playing Tetris did not change participants' retrospective evaluation of their worst craving experience, measured using the $C E Q$, but this craving would not necessarily have coincided with one of the seven daily prompts to play Tetris. Future research should test the effectiveness of Tetris in field settings when used spontaneously. 
In conclusion, playing Tetris weakened cravings for a wide range of addictive and everyday substances and activities, and did so consistently over the week-long study period. As a support tool, Tetris could help people manage their cravings in naturalistic settings and over extended time periods. This finding is consistent with theories such as El Theory (Kavanagh et al., 2005) that view cravings as conscious states supported by limited capacity cognitive processes. Laboratory research on the effects of modalitycongruent cognitive loads on craving has focused primarily on food cravings. The present study shows for the first time that this research generalizes to cravings for addictive substances, and activities that do not involve consumption, in naturalistic settings. 


\section{References}

Andrade, J., Pears, S., May, J., \& Kavanagh, D.J. (2012) Use of a clay modeling task to reduce chocolate craving. Appetite, 58, 955-963. Doi: 10.1016/j.appet.2012.02.044

American Psychiatric Association (2013). Diagnostic and statistical manual of mental disorders (5th ed.). Arlington, VA: APA.

Baddeley, A., \& Andrade, J. (2000). Working memory and the vividness of imagery. Journal of Experimental Psychology: General, 129, 126-145

Burton, S. M., \& Tiffany, S. T. (1997). The effect of alcohol consumption on imaginal and in vivo manipulations of smoking urges. Addiction, 92, 15-26

Connor, J.P., Kavanagh, D.J., Andrade, J., May, J., Feeney, G.F.X, Gullo, M.J., White, A.M., Fry, M-L., Drennan, J., Previte, J., Tjondronegoro, D. (2014). Alcohol consumption in young adults: the role of multisensory imagery. Addictive Behaviors, 39, 721-724. doi: /10.1016/j.addbeh.2013.11.023

Gammage, K. L., Hall, C. R., \& Rodgers, W. M. (2000). More about exercise imagery. Sport psychologist, 14(4), 348-359.

Hall, C. R., Rodgers, W. M., Wilson, P. M., \& Norman, P. (2010). Imagery Use and SelfDetermined Motivations in a Community Sample of Exercisers and Non Exercisers1. Journal of Applied Social Psychology, 40(1), 135-152.

Harvey, K., Kemps, E. \& Tiggemann, M, (2005). The nature of imagery processes underlying food cravings. British Journal of Health Psychology, 10, 49-56

Hofmann, W., Baumeister R., F., Förster, G. \& Vohs, K., D. (2011). Everyday temptations: an experience sampling study of desire, conflict, and self-control. Journal of Personality and Social Psychology, 102(6), 1318-1335

Holmes, E. A., James, E. L., Coode-Bate, T., \& Deeprose, C. (2009). Can playing the computer game 'Tetris' reduce the build-up of flashbacks for trauma? A proposal from cognitive science. PLOS ONE, 4, 1.

Holmes, E. A., James, E. L., Kilford, E. J., \& Deeprose, C. (2010). Key steps in developing a cognitive vaccine against traumatic flashbacks: visuospatial Tetris versus verbal Pub Quiz. PloS one, 5(11), e13706.

Hsu, A, Yang, J, Yilmaz, Y, Haque, MS, Can, C, Blandford, A. (2014). Persuasive technology for overcoming food cravings and improving snack choices. Proceedings of the SIGCHI Conference on Human Factors in Computing Systems. New York: ACM. Pp. 3403-3412

Kavanagh, D.J., May, J. \& Andrade, J. (2009) Tests of the elaborated intrusion theory of craving and desire: Features of alcohol craving during treatment for an alcohol 
disorder. British Journal of Clinical Psychology, 48, 251-254. doi: $10.1348 / 014466508 \times 387071$

Kavanagh, D.J., Andrade, J., \& May, J. (2005) Imaginary relish and exquisite torture: The elaborated intrusion theory of desire. Psychological Review, 112, 446-467. doi: 10.1037/0033-295X.112.2.446

Kemps, E., \& Tiggeman, M. (2007). Modality-specific imagery reduces cravings for food: An application of the Elaborated Intrusion Theory of Desire to food craving. Journal of Experimental Psychology: Applied, 13, 95-104

Kemps, E, Tiggemann, M. (2009). Competing visual and olfactory imagery tasks suppress craving for coffee. Experimental and Clinical Psychopharmacology, 17(1), 43-50.

Kemps, E, Tiggemann, M (2013). Hand-held dynamic visual noise reduces naturally occurring food cravings and craving-related consumption. Appetite, 68, 152-157.

Kemps, E., Tiggemann, M., \& Christianson, R. (2008). Concurrent visuo-spatial processing reduces food cravings in prescribed weight-loss dieters. Journal of Behavior Therapy and Experimental Psychiatry, 39, 177-186.

Knäuper, B., Pillay, R., Lacaille, J., McCollam, A. \& Kelso, E. (2011). Replacing craving imagery with alternative pleasant imagery reduces craving intensity. Appetite, 57, 173-178.

May, J., Andrade, J., Kavanagh, D.K., Feeney, G.F.X., Gullo, M.J., Statham, D., SkorkaBrown, J., Cassimatis, M., Young, R.McD., Connor, J.P. (2014) The Craving Experience Questionnaire: A Brief, Theory-Based Measure of Consummatory Desire and Craving. Addiction, 109, 728-735. doi:10.1111/add.12472.

May, J., Andrade, J., Kavanagh, D.J. \& Penfound, L. (2008) Imagery and strength of craving for eating, drinking and playing sport. Cognition and Emotion, 22, 633-650. doi:10.1080/02699930701446296

May, J., Andrade, J., Panabokke, N. \& Kavanagh, D.J. (2004) Images of Desire: Cognitive Models of Craving. Memory, 12(4), 447-461. doi: 10.1080/09658210444000061

May, J., Andrade, J., Panabokke, N. \& Kavanagh, D.J. (2010) Visuospatial tasks suppress craving for cigarettes. Behaviour Research and Therapy, 48, 476-485. doi: 10.1016/j.brat.2010.02.001

May, J., Andrade, J., Willoughby, K. \& Brown, C. (2012) An attentional control task reduces intrusive thoughts about smoking. Nicotine \& Tobacco Research, 14(4), 472-478. doi: $10.1093 /$ ntr/ntr238 
Piasecki , T. M. , Richardson , A. E. , \& Smith , S. M. ( 2007 ). Self-monitored motives for smoking among college students. Psychology of Addictive Behaviors , 21 , 328-337.

Salkovskis, P. M., \& Reynolds, M. (1994). Thought suppression and smoking cessation. Behaviour research and therapy, 32(2), 193-201.

Shiffman, S., Dunbar, M. S., Kirchner, T. R., Li, X., Tindle, H. A., Anderson, S. J., ... \& Ferguson, S. G. (2013). Cue reactivity in non-daily smokers.

Psychopharmacology, 226(2), 321-333.

Shiffman S, Gwaltney CJ, Balabanis M, Liu KS, Paty JA, Kassel JD, Hickcox M, Gnys M (2002) Immediate antecedents of cigarette smoking: an analysis from ecological momentary assessment. Journal of Abnormal Psychology, 111, 531-545

Shiffman, S., Engberg, J. B., Paty, J. A., Perz, W. G., Gnys, M., Kassel, J. D., \& Hickcox, M. (1997). A day at a time: predicting smoking lapse from daily urge. Journal of Abnormal Psychology, 106(1), 104-116.

Skorka-Brown, J., Andrade, J., \& May, J., (2014) Playing 'Tetris' reduces the strength, frequency and vividness of naturally occurring cravings Appetite, 76, 161-165. doi: 10.1016/j.appet.2014.01.073)

StataCorp. (2013). Stata Statistical Software: Release 13. College Station, TX: StataCorp LP.

Statham, D. J., Connor, J. P., Kavanagh, D. J., Feeney, G. F. X., Young, R. McD., May, J., \& Andrade, J. (2011). Measuring Alcohol Craving: Development of the Alcohol Craving Questionnaire. Addiction 106(7), 1230-1238. doi: 10.1111/j.13600443.2011.03442.x

Tiffany, S. T. and Wray, J. M. (2012), The clinical significance of drug craving. Annals of the New York Academy of Sciences, 1248, 1-17. doi: 10.1111/j.17496632.2011.06298.x

Van Dillen, L. F., Papies, E. K., \& Hofmann, W. (2013). Turning a blind eye to temptation: How cognitive load can facilitate self-regulation. Journal of Personality and Social

Versland, A \& Rosenberg, H. (2007). Effect of brief imagery interventions on craving in college student smokers. Addiction Research and Theory, 15(2), 177-187

Waters, A. J., \& Li, Y. (2008). Evaluating the utility of administering a reaction time task in an ecological momentary assessment study. Psychopharmacology,197(1), 2535.

World Health Organization. (1992). The ICD-10 Classification of Mental and Behavioural Disorders: Clinical Descriptions and Diagnostic Guidelines. WHO Press. Geneva 
Wray, J.M., Gass, J.C., \& Tiffany, S.T. (2013). A systematic review of the relationships between craving and smoking cessation. Nicotine Tobacco Research, 15(7), 11671182. 